OPEN ACCESS

Edited by:

Jia Yin,

Hunan Normal University, China

Reviewed by:

Shiyu Tao,

Huazhong Agricultural University,

China

Tarique Hussain

Nuclear Institute for Agriculture and Biology, Pakistan

Dandan Han,

China Agricultural University, China

${ }^{*}$ Correspondence: Xiaokang Ma

maxiaokang@hunau.edu.cn

Specialty section:

This article was submitted to

Microbial Symbioses,

a section of the journal

Frontiers in Microbiology

Received: 01 September 2021

Accepted: 23 September 2021

Published: 29 October 2021

Citation:

Li ZQ, Tang LZ, Liu N, Zhang F

Liu X, Jiang $Q$, Chen JS and Ma XK

(2021) Comparative Effects

of Compound Enzyme and Antibiotics

on Growth Performance, Nutrient Digestibility, Blood Biochemical Index, and Intestinal Health in Weaned Pigs.

Front. Microbiol. 12:768767.

doi: 10.3389/fmicb.2021.768767

\section{Comparative Effects of Compound Enzyme and Antibiotics on Growth Performance, Nutrient Digestibility, Blood Biochemical Index, and Intestinal Health in Weaned Pigs}

\author{
Zhiqing Li, Lizi Tang, Nian Liu, Fan Zhang, Xiang Liu, Qian Jiang, Jiashun Chen and \\ Xiaokang Ma*
}

College of Animal Science and Technology, Hunan Agricultural University, Changsha, China

This experiment aims to explore the effects of compound enzyme preparation substituting chlortetracycline on growth performance, serum immune markers, and antioxidant capacity and intestinal health in weaned piglets. A total of twenty-four 28day-old "Duroc $\times$ Landrace $\times$ Yorkshire" weaned piglets with an average initial weight of $7.25 \pm 0.25 \mathrm{~kg}$ were randomly divided into three groups according to their body weight, with eight replicates in each group and one pig in each replicate. The three dietary treatments were basal diet (CON), basal diet $+1,000 \mathrm{mg} / \mathrm{kg}$ compound enzyme preparation (cellulase 4,000 $\mathrm{IU} / \mathrm{g}, \alpha$-amylase 1,000 $\mathrm{lU} / \mathrm{g}, \beta$-glucanase $150 \mathrm{lU} / \mathrm{g}$, and neutral protease 3,000 IU/g, CE), and basal diet $+75 \mathrm{mg} / \mathrm{kg}$ chlortetracycline (CTC). The animal experiment lasted for 28 days and was divided into two stages: the early stage (0-14 days) and the late stage (15-28 days). The results showed that (1) compared with the CON, the CE and CTC significantly increased the ADG of weaned piglets during the early and whole period of experiment $(p<0.05)$, decreased the F:G in the whole experiment period $(p<0.05)$, and diarrhea rate in the early stage $(p<0.01)$. (2) Compared with the CON, the apparent total tract digestibility of ADF and NDF was significantly increased in pigs fed the CE diet in the early and late stages of experiment $(p<0.05)$ with no significant difference compared with the CTC. (3) Compared with the $\mathrm{CON}$, the concentrations of serum IgA and SOD in weaned piglets were significantly increased in the CE group in the early stage of the experiment $(p<0.05)$. (4) Compared with the CON group, the acetic acid, propionic acid, and total VFA contents in cecum and colon segments were elevated in the CE group $(0<0.05)$ with no significant difference compared with the CTC. (5) Compared with the CON group, the villus height of duodenum and jejunum and the ratio of villus height to recess depth of ileum were increased in the CE and CTC group $(p<0.05)$. (6) Compared with the CON group, the abundance of Lactobacillus significantly increased $(p<0.01)$ while the abundance of 
Escherichia coli decreased in the CE group and CTC group ( $p<0.01)$. In conclusion, CE preparation instead of CTC can significantly improve the nutrient digestibility, the immunity, antioxidant capacity, and intestinal health of pigs, which may contribute to the improved growth performance of piglets.

Keywords: compound enzymes, growth performance, nutrient digestibility, serum biochemical profiles, intestinal health, weaned piglets

\section{INTRODUCTION}

In the pig industry, weaning stress of piglets has always been a huge problem. Piglets have a series of problems such as diarrhea, anorexia, and decreased growth performance due to immature digestive tract, low immune function, and dietary changes ( $\mathrm{Li}$ et al., 2021). In the past, antibiotics were commonly used in piglets to reduce weaning stress, but bacterial resistance to antibiotics has become a global threat to animals and public health (Teale and Borriello, 2021). The inclusion of antibiotics in animal diets is a controversial issue worldwide (Zainab et al., 2020). China stopped using antibiotics as feed additives in July 2020. Therefore, it is necessary to develop new feed additives to replace antibiotics and improve the health condition of pigs and its product quality.

Since antibiotics were banned, enzymes used in pig diets have been widely accepted (Willamil et al., 2012). It has been reported that dietary supplementation of compound enzyme containing amylase, protease, and xylanase can promote the growth of piglets by improving nutrient digestibility and regulating intestinal flora (Yi et al., 2013). Since cereal feeds contain large amounts of soluble non-starch polysaccharides (NSP), it not only reduced nutrient digestibility but also induced inflammation in pigs (Woyengo et al., 2009; Vila et al., 2018). Moreover, starch particles embedded in cereal protein matrix are not easily decomposed by starch degrading enzymes (Zaefarian et al., 2015). The adverse effects of NSP and cellulose could be alleviated by adding cellulase, $\alpha$-amylase, and $\beta$-glucanase to cereal grain-based diets. In addition, supplementation of cereal grain-based diets with protease for pigs may increase the degradation of protein that interacts with NSP and starch, thereby increasing nutrient digestibility.

At present, most studies mainly explore the effects of different enzyme preparations on barley, oats, or Distillers Dried Grains with Solubles (DDGS) as the basic diet, while there are few studies investigating whether enzyme preparations can improve the utilization efficiency of corn-soybean meal diet. Therefore, this experiment was conducted to evaluate the effects of adding a compound enzyme preparation composed of NSP enzyme,

Abbreviations: BW, body weight; ADG, average daily gain; ADFI, average daily feed intake; G:F, gain-to-feed ratio; DM, dry matter; OM, organic matter; CP, crude protein; GE, gross energy; ADF, acid detergent fiber; NDF, neutral detergent fiber; IgA, immunoglobulin A; IgG, immunoglobulin G; IgM, immunoglobulin M; SOD, superoxide dismutase; GSH-Px, glutathione peroxidase; T-AOC, total antioxidant capacity; MDA, malondialdehyde; VFAs, volatile fatty acids; SCFAs, short-chain fatty acids; BCFAs, branched-chain fatty acids; PCR, polymerase chain reaction; OTUs, operational taxonomic units; PCoA, principal coordinate analysis; LDA, linear discriminant analysis; LEfSe, linear discriminant analysis effect size. amylase and protease to replace antibiotics in corn-soybean meal diet on growth performance, serum immune markers, and antioxidant capacity and intestinal health of weaned piglets. It provides scientific basis and theoretical basis for the application of compound enzyme preparation to replace antibiotics in animal production.

\section{MATERIALS AND METHODS}

The compound enzyme used in this study contained amylase, protease, and xylanase. The compound enzyme preparation is provided by DuPont, Inc. The main components are cellulase (4,000 IU/g), $\alpha$-amylase (1,000 IU/g), $\beta$-glucanase (150 IU/g), and neutral protease $(3,000 \mathrm{IU} / \mathrm{g})$. According to the results of in vitro test (Long et al., 2021) and the company's recommendation, the supplemental amount was $1,000 \mathrm{mg} / \mathrm{kg}$.

\section{Animal Treatment and Experimental Design}

Twenty-four 28-day-old weaned piglets (Duroc $\times$ Landrace $\times$ Large White) with an average initial body weight of $7.25 \mathrm{~kg}$ were randomly allocated to three groups with eight replicates per group and one piglet per replicate according to gender and body weight in a randomized complete block design. The three experimental diets were a corn-soybean meal basal diet $(\mathrm{CON})$, a basal diet supplemented with $1,000 \mathrm{mg} / \mathrm{kg}$ compound enzyme preparation (CE) and a basal diet supplemented with $75 \mathrm{mg} / \mathrm{kg}$ chlorotetracycline preparation (CTC). The animal experiment lasted for 28 days, which was divided into early (0-14 days) and late (15-28 days) stages. The nutrient composition of diets (Table 1) met or exceeded the requirements of NRC (2012).

These experiments were conducted in accordance with Chinese guidelines for animal welfare and experimental protocols, and all animal procedures were approved by the Committee of Animal Care at Hunan Agricultural University (Changsha, China) (Permit Number: CACAHU 2021-01106). The animal experiments were carried out in the Animal Test Base of Hunan Agricultural University. All the piglets were housed on a net bed with a leaky floor and had free access to feed and water. The piglets were dewormed and immunized according to routine procedures and pig houses were cleaned and disinfected regularly. The ambient temperature is automatically adjusted by the thermostatic controller and windows are regularly opened for ventilation. 


\section{DETECTION INDICATORS}

\section{Growth Performance and Diarrhea Rate}

On days 0,14 , and 28, the body weight and feed intake of pigs were recorded to calculate average daily gain (ADG), average daily feed intake (ADFI), and feed to weight gain ratio (F:G). From days 0 to 28, the health status and mortality of each piglet were recorded. The occurrence of diarrhea and fecal score of each pig was visually assessed and evaluated twice a day by trained observers blinded to the treatments according to the method of Long et al. (2017). In brief, the severity of diarrhea was assessed using a scoring system as shown below: $1=$ hard stool; 2 = slightly soft feces; 3 = partially formed soft feces; 4 = loose semi-liquid feces; 5 = watery mucous feces. Pigs are identified as having diarrhea when the average score exceeds 3 points. Diarrhea rate was determined mainly based on the average score of feces and the formula was: diarrhea rate $(\%)=$ days of diarrhea number of pigs with diarrhea/(test days $\times$ total number of pigs).

\section{Nutrient Digestibility}

During this experiment, a total of $1 \mathrm{~kg}$ of feed samples were collected weekly. The dry matter (DM), crude protein (CP), acid detergent fiber (ADF), and neutral detergent fiber (NDF) in feed and fecal samples were measured according to AOAC (2012). The apparent total tract digestibility (ATTD) was calculated as follows:

$$
\begin{aligned}
& \text { ATTD nutrient }=1-\left(\mathrm{Cr}_{\text {diet }} \cdot \text { Nutrient }_{\text {feces }}\right) / \\
& \left(C r_{\text {feces }} \cdot \text { Nutrient }_{\text {diet }}\right) \text {. }
\end{aligned}
$$

\section{Serum Assays}

On days 14 and 28, blood samples were collected by anterior vena cava puncture in each treatment, centrifuged at $3,000 \mathrm{~g}$ at $4^{\circ} \mathrm{C}$ for $15 \mathrm{~min}$ to obtain the serum, and stored at $-20^{\circ} \mathrm{C}$ until analysis. Serum immunoglobulin concentration was determined by using an ELISA kit (A) following the manufacturer's instructions (Cusabio Biotechnology Co., Ltd., Wuhan, China). Moreover, the contents of total antioxidant capacity (T-AOC), superoxide dismutase (SOD), glutathione peroxidase (GSH-Px), and malondialdehyde (MDA) in serum were determined using a spectrophotometer (Leng Guang SFZ1606017568, Shanghai, China) following the manufacturer's instructions (Nanjing Jiancheng Bioengineering Institute, Nanjing, China).

\section{Volatile Fatty Acids Analysis}

Digesta samples from cecal, colonic, and ileum segments were collected on day 28 to determine volatile fatty acid (VFA) content $(n=8)$. All samples were frozen in a $-80^{\circ} \mathrm{C}$ freezer immediately after collection. VFA content of digesta was determined using a HP 5, 890 gas chromatograph (HP, Pennsylvania, United States) according to the method of Long et al. (2017).

\section{Morphology of Small Intestine}

At the end of the animal experiment, 24 pigs (8 pigs per treatment) were slaughtered and the proximal, middle, and distal part of the small intestine from the gastric pylorus to the ileo-cecal valve were obtained to analyze the morphological changes of duodenum, jejunum, and ileum. The samples of small intestine were fixed in neutral-buffered formalin and processed by the standard paraffin method. Small intestine sections $(9-10 \mathrm{~cm})$ were stained with hematoxylin and eosin. Measurements of villus height and crypt depth were taken only from sections where the plane of section ran vertically from the tip of villus to the base of an adjacent crypt by using a light microscope. Ten well-oriented villus $\times 3$ sections of each pig were used to determine these indices (Wang et al., 2021).

\section{Microbiota Analysis by 16S RNA}

Total genome DNA was extracted from cecal digesta samples using the QIAamp Fast DNA Stool mini kit (Qiagen, Hilden, Germany) and checked with $1 \%$ agarose gel. The DNA concentration and purity were determined with Nano Drop 2,000 UV-vis spectrophotometer (Thermo Fisher Scientific, Wilmington, United States). The specific primer with the barcode

TABLE 1 | Ingredient composition and nutrient levels of the experimental diets

\begin{tabular}{|c|c|}
\hline Items & Content (\%) \\
\hline \multicolumn{2}{|l|}{ Ingredients } \\
\hline Corn & 54.75 \\
\hline Soybean meal & 19.00 \\
\hline Full-fat soybean powder & 10.00 \\
\hline Fish meal & 5.00 \\
\hline Whey powder & 6.15 \\
\hline Soybean oil & 1.50 \\
\hline Dicalcium phosphate & 0.90 \\
\hline L-Lysine- $\mathrm{HCl}$ & 0.48 \\
\hline L-Threonine & 0.05 \\
\hline DL-Methionine & 0.10 \\
\hline L-Tryptophan & 0.02 \\
\hline Salt & 0.30 \\
\hline Limestone & 0.50 \\
\hline Premix $^{a}$ & 1.00 \\
\hline $\mathrm{Cr}_{2} \mathrm{O}_{3}$ & 0.25 \\
\hline Total & 100.00 \\
\hline \multicolumn{2}{|l|}{ Calculated nutrients } \\
\hline Digestible energy (MJ/kg) & 14.64 \\
\hline Crude protein & 20.15 \\
\hline Lysine & 1.38 \\
\hline Methionine & 0.82 \\
\hline Methionine + cysteine & 1.01 \\
\hline Threonine & 0.97 \\
\hline Tryptophan & 0.25 \\
\hline Calcium & 0.80 \\
\hline Total phosphorus & 0.73 \\
\hline
\end{tabular}
(\%, as-fed basis).

aThe premix provided the following (per kilogram of complex feed): Vitamin A, 12,000 U; Vitamin D, 2,500 U; Vitamin E, $30 \mathrm{U}$; Vitamin B12, $12 \mu \mathrm{g}$; Vitamin K, $3 \mathrm{mg}$; d-pantothenic acid, $15 \mathrm{mg}$; nicotinic acid, $40 \mathrm{mg}$; choline chloride, 400 mg; Mn, 40 mg; Zn, 100 mg; Fe, 90 mg; Cu, 8.8 mg; l, 0.35 mg; Se, 0.3 mg. 
(16S V3-V4) were amplified by an ABI Gene Amp ${ }^{\circledast}$ 9,700 PCR thermocycler (ABI, CA, United States). Then, the PCR products were extracted, purified, and quantified.

Paired-end sequencing was performed on an Illumina MiSeq PE300 platform/NovaSeq PE250 platform (Illumina, San Diego, United States). The raw 16S rRNA gene sequencing reads were demultiplexed, quality-filtered and merged according to previous studies (Magoč and Salzberg, 2011; Chen et al., 2018). The complexity of species diversity was evaluated with ACE and Chao richness estimators and diversity indices of Shannon and Simpson (Edgar, 2013). $\beta$-diversity was evaluated using principal component analysis (PCA) based on the Euclid distance. The significant differences between samples were evaluated by analysis of similarities (ANOSIM).

OTUs representing $<0.005 \%$ of the population were removed and taxonomy was assigned using the RDP classifier. The relative abundance of each OTU was counted at different taxonomic levels. Then, bioinformatics analysis was mainly performed using QIIME (v1.7.0) and R packages (v3.2.0). The OTU table in QIIME was used to calculate OTU-level and $\beta$-diversity was assessed by principal coordinate analysis ( $\mathrm{PCoA})$. Cluster analysis and significant differences between samples were tested by ANOSIM.

\section{Statistical Analysis}

Differences in the diarrhea incidence were tested by the $\chi^{2}$ contingency test. All other data were analyzed by ANOVA method using the GLM model in SAS 9.2 statistical software with repetition (cycle) as the statistical unit. A very significant difference between the means was defined as $p \leq 0.01$ and significant difference between the means was defined at $p \leq 0.05$, while a trend for the significance between the means was designated at $0.05<p \leq 0.10$.

\section{RESULTS}

\section{Growth Performance and Diarrhea Rate}

As shown in Table 2, pigs in the CE group had higher ADG during days $0-14$ and days $0-28$ and higher body weight at days 14 and 28 than pigs in the CON group $(p<0.05)$ without significant difference compared with the CTC. The CE diet did not significantly change the ADFI of pigs, but in the early stage and the whole period of the experiment, the F:G ratio and the diarrhea rate from days 0 to 14 were significantly reduced compared with the CON diet $(p<0.05)$. However, there was no differences in growth performance or diarrhea rate between CE and CTC.

\section{Nutrient Digestibility}

The effects of $\mathrm{CE}$ on nutrients in weaned piglets are shown in Table 3. Compared with the CON, the apparent total tract digestibility of ADF and NDF was significantly increased in pigs fed CE diet in the early and late stages of experiment $(p<0.05)$, and there was no significant difference between the CE and CTC groups.

\section{Immunity and Antioxidant Properties}

As shown in Table 4 that compared with the CON, CE induced higher serum IgA concentrations in weaned piglets on day 14 $(p<0.05)$ and higher IgG on day 28 , which was not significantly different from the CTC. Table 5 shows that pigs fed CE had higher serum SOD concentration on day $14(p<0.05)$ compared with the CON and was not significantly different from the CTC. The CE group showed increased serum GSH-Px concentration of pigs on day 28 compared with the CON $(p<0.05)$ without

TABLE 2 | Effect of CE on growth performance and diarrhea rate of weaned piglets.

\begin{tabular}{lccccc}
\hline Items & CON & CE & CTC & SEM & p-value \\
\hline Day 0 BW (kg) & 7.25 & 7.24 & 7.25 & 0.04 & 0.97 \\
Day 14 BW (kg) & $10.97^{\mathrm{b}}$ & $11.45^{\mathrm{a}}$ & $11.34^{\mathrm{a}}$ & 0.12 & 0.03 \\
Day 28 BW (kg) & $15.88^{\mathrm{b}}$ & $16.80^{\mathrm{a}}$ & $16.66^{\mathrm{a}}$ & 0.21 & 0.01 \\
Days 0-14 & & & & & \\
ADG (g) & $265.71^{\mathrm{b}}$ & $300.98^{\mathrm{a}}$ & $292.14^{\mathrm{a}}$ & 7.54 & 0.01 \\
ADFI (g) & 441.43 & 456.09 & 460.01 & 9.33 & 0.20 \\
F:G & $1.66^{\mathrm{a}}$ & $1.51^{\mathrm{b}}$ & $1.57^{\mathrm{b}}$ & 0.02 & $<0.01$ \\
Diarrhea rate (\%) & $3.46^{\mathrm{a}}$ & $1.34^{\mathrm{b}}$ & $1.23^{\mathrm{b}}$ & 0.39 & $<.01$ \\
Days 15-28 & & & & & 0.39 \\
ADG (g) & 351.07 & 382.32 & 379.91 & 17.24 & 0.97 \\
ADFI (g) & 658.21 & 655.27 & 660.45 & 15.47 & 0.97 \\
F:G & 1.89 & 1.73 & 1.74 & 0.06 & 0.13 \\
Diarrhea rate (\%) & 1.00 & 1.00 & 1.23 & 0.57 & 0.95 \\
Days 0-28 & & & & & 0.013 \\
ADG (g) & $308.39^{\mathrm{b}}$ & $341.65^{\mathrm{a}}$ & $336.03^{\mathrm{a}}$ & 7.52 & 0.02 \\
ADFI (g) & 549.82 & 560.18 & 560.22 & 5.30 & 0.31 \\
F:G & $1.79^{\mathrm{a}}$ & $1.65^{\mathrm{b}}$ & $1.67^{\mathrm{b}}$ & 0.03 & 0.01 \\
Diarrhea rate (\%) & 2.23 & 1.17 & 1.23 & 0.39 & 0.13 \\
\hline SEM standard error & & & & &
\end{tabular}

SEM, standard error of the mean $(n=8)$.

${ }^{a, b}$ Different superscripts within a row indicate a significant difference $(p<0.05)$.

TABLE 3 | Effects of CE on total intestinal apparent digestibility of nutrients in weaned piglets (\%).

\begin{tabular}{llllll}
\hline Items & CON & CE & CTC & SEM & p-value \\
\hline Days 0-14 & & & & & \\
DM & 81.51 & 81.16 & 82.01 & 0.40 & 0.34 \\
OM & 83.93 & 83.18 & 83.53 & 0.33 & 0.31 \\
CP & 76.14 & 75.12 & 76.87 & 0.55 & 0.12 \\
GE & 81.61 & 81.11 & 82.08 & 0.32 & 0.13 \\
ADF & $56.82^{\mathrm{b}}$ & $65.20^{\mathrm{a}}$ & $65.99^{\mathrm{a}}$ & 1.75 & $<0.01$ \\
NDF & $40.92^{\mathrm{b}}$ & $54.74^{\mathrm{a}}$ & $51.05^{\mathrm{a}}$ & 2.44 & $<0.01$ \\
Days 15-28 & & & & & \\
DM & 81.17 & 80.36 & 80.58 & 0.27 & 0.13 \\
OM & 83.29 & 82.72 & 82.54 & 0.29 & 0.19 \\
CP & 75.19 & 76.31 & 76.22 & 0.57 & 0.33 \\
GE & 81.28 & 80.39 & 80.46 & 0.35 & 0.17 \\
ADF & $59.81^{\mathrm{b}}$ & $64.38^{\mathrm{a}}$ & $64.44^{\mathrm{a}}$ & 1.25 & 0.03 \\
NDF & $46.90^{\mathrm{b}}$ & $54.86^{\mathrm{a}}$ & $55.87^{\mathrm{a}}$ & 2.01 & 0.01 \\
\hline
\end{tabular}

SEM, standard error of the mean $(n=8)$.

${ }^{a, b}$ Different superscripts within a row indicate a significant difference $(p<0.05)$. 
significant difference compared with the CTC group. Moreover, the CE diet tended to decrease the serum MDA concentration in weaned piglets compared with the CON $\operatorname{diet}(0.05<p \leq 0.10)$.

\section{Volatile Fatty Acid Composition}

VFA profiles of ileal, cecal, and colonic segments are shown in Table 6. Pigs fed CE diet had increased acetic acid, propionic acid, and total VFA contents in the cecum and colon compared with $\mathrm{CON}(p<0.05)$ with no significant difference compared with the CTC.

\section{Intestinal Morphology}

As shown in Table 7, pigs in CE group had lower crypt depth and higher villus height and villus height-to-crypt depth ratio in jejunum compared with that of CON $(p<0.01)$. The duodenum villus height of pigs in the CE group was significantly higher than that in the CON $(p<0.05)$ with no significant difference compared with the CTC.

\section{Cecal Microbiota}

Venn diagrams showed that there were 684, 543, and 755 OTUs in cecal digesta samples of CE, CON, and CTC groups, respectively, of which 423 OTUs were shared and 201 OTUs were unique (Figure 1A). The $\beta$-diversity of bacterial community

TABLE 4 | Effects of dietary CE supplementation on serum immunological traits of weaned pigs.

\begin{tabular}{llllll}
\hline Items & CON & CE & CTC & SEM & $\boldsymbol{p}$-value \\
\hline Day 14 & & & & & \\
$\operatorname{lgA}(\mathrm{g} / \mathrm{L})$ & $1.00^{\mathrm{b}}$ & $1.17^{\mathrm{a}}$ & $1.14^{\mathrm{a}}$ & 0.04 & 0.01 \\
$\operatorname{lgG}(\mathrm{g} / \mathrm{L})$ & 6.93 & 7.36 & 7.99 & 0.33 & 0.11 \\
$\lg \mathrm{g}(\mathrm{g} / \mathrm{L})$ & 0.57 & 0.57 & 0.64 & 0.02 & 0.09 \\
$\operatorname{Day} \mathbf{2 8}$ & & & & & \\
$\operatorname{lgA}(\mathrm{g} / \mathrm{L})$ & 2.81 & 2.59 & 2.73 & 0.08 & 0.23 \\
$\operatorname{lgG}(\mathrm{g} / \mathrm{L})$ & $8.34^{\mathrm{b}}$ & $9.73^{\mathrm{a}}$ & $9.85^{\mathrm{a}}$ & 0.40 & 0.03 \\
$\operatorname{lgM}(\mathrm{g} / \mathrm{L})$ & 0.10 & 0.11 & 0.12 & 0.01 & 0.16 \\
\hline
\end{tabular}

SEM, standard error of the mean $(n=8)$.

$a, b$ Different superscripts within a row indicate a significant difference $(p<0.05)$.

TABLE 5 | Effect of CE on antioxidant parameters of weaned piglets.

\begin{tabular}{lrrrrr}
\hline Items & CON & CE & CTC & SEM & $\boldsymbol{p}$-value \\
\hline Day 14 & & & & & \\
GSH-Px $(\mathrm{U} / \mathrm{ml})$ & 748.05 & 752.22 & 768.75 & 24.47 & 0.82 \\
MDA $(\mathrm{nmol} / \mathrm{ml})$ & 4.02 & 3.32 & 2.87 & 0.30 & 0.05 \\
SOD $(\mathrm{U} / \mathrm{ml})$ & $119.78^{\mathrm{b}}$ & $133.13^{\mathrm{a}}$ & $140.16^{\mathrm{a}}$ & 4.03 & $<.01$ \\
T-AOC $(\mathrm{U} / \mathrm{ml})$ & 8.48 & 9.40 & 10.11 & 0.62 & 0.21 \\
Day 28 & & & & & \\
GSH-Px $(\mathrm{U} / \mathrm{ml})$ & $297.68^{\mathrm{b}}$ & $319.53^{\mathrm{a}}$ & $320.37^{\mathrm{a}}$ & 5.44 & 0.02 \\
MDA $(\mathrm{nmol} / \mathrm{ml})$ & 5.84 & 4.38 & 4.28 & 0.49 & 0.07 \\
SOD $(\mathrm{U} / \mathrm{ml})$ & 180.43 & 180.87 & 180.29 & 5.70 & 0.76 \\
T-AOC $(\mathrm{U} / \mathrm{ml})$ & 7.75 & 7.80 & 8.08 & 0.34 & 0.76
\end{tabular}

SEM, standard error of the mean $(n=8)$.

${ }^{a, b}$ Different superscripts within a row indicate a significant difference $(p<0.05)$.
TABLE 6 | Effect of CE on VFA content in different intestinal segments of weaned piglets $(\mathrm{mg} / \mathrm{kg})$.

\begin{tabular}{|c|c|c|c|c|c|}
\hline Items & CON & CE & стс & SEM & $p$-value \\
\hline \multicolumn{6}{|l|}{ Ileum } \\
\hline Acetic acid & 489.06 & 481.36 & 491.70 & 43.59 & 0.98 \\
\hline Propionic acid & 129.42 & 136.85 & 144.32 & 16.67 & 0.82 \\
\hline Isobutyric acid & 16.98 & 17.45 & 15.97 & 0.68 & 0.32 \\
\hline Butyric acid & 62.86 & 68.16 & 69.00 & 5.70 & 0.72 \\
\hline Isovaleric acid & 7.13 & 8.83 & 7.49 & 1.45 & 0.69 \\
\hline Valeric acid & 8.09 & 8.24 & 8.91 & 0.80 & 0.74 \\
\hline Total VFA & 713.51 & 720.89 & 737.45 & 44.56 & 0.93 \\
\hline \multicolumn{6}{|l|}{ Caecum } \\
\hline Acetic acid & $3,433.76^{b}$ & $4,514.55^{a}$ & $4,449.69^{a}$ & 309.32 & 0.04 \\
\hline Propionic acid & $2,014.41^{b}$ & $2,792.03^{a}$ & $2,809.36^{a}$ & 223.22 & 0.04 \\
\hline Isobutyric acid & 82.18 & 55.62 & 91.19 & 13.09 & 0.17 \\
\hline Butyric acid & $1,056.40$ & $1,194.49$ & $1,233.27$ & 94.99 & 0.41 \\
\hline Isovaleric acid & 90.29 & 70.91 & 90.73 & 9.92 & 0.30 \\
\hline Valeric acid & 193.99 & 180.81 & 210.94 & 22.67 & 0.65 \\
\hline Total VFA & $6,871.04^{b}$ & $8,808.40^{a}$ & $8,885.18^{a}$ & 564.34 & 0.04 \\
\hline \multicolumn{6}{|l|}{ Colon } \\
\hline Acetic acid & $2,538.82^{b}$ & $3,141.48^{a}$ & $3,305.58^{a}$ & 186.45 & 0.03 \\
\hline Propionic acid & $1,616.77^{b}$ & $2,083.41^{a}$ & $2,207.99^{a}$ & 140.07 & 0.02 \\
\hline Isobutyric acid & 90.24 & 120.99 & 113.61 & 12.31 & 0.22 \\
\hline Butyric acid & $1,142.52$ & $1,222.97$ & 1, 295.80 & 118.56 & 0.67 \\
\hline Isovaleric acid & 161.77 & 192.08 & 185.77 & 11.10 & 0.16 \\
\hline Valeric acid & 306.73 & 327.35 & 300.58 & 22.84 & 0.69 \\
\hline Total VFA & $5,856.84^{b}$ & $7,088.28^{a}$ & $7,409.33^{\mathrm{a}}$ & 326.98 & 0.01 \\
\hline
\end{tabular}

SEM, standard error of the mean $(n=8)$.

${ }^{a, b}$ Different superscripts within a row indicate a significant difference $(p<0.05)$.

Total VFAs $=$ Acetate + Propionate + Butyrate + Valerate + Isobutyrate + Isovalerate.

TABLE 7 | Effect of CE on intestinal morphology of weaned piglets.

\begin{tabular}{|c|c|c|c|c|c|}
\hline Items & CON & CE & СтC & SEM & $p$-value \\
\hline \multicolumn{6}{|l|}{ Duodenum } \\
\hline Villus height ( $\mu \mathrm{m})$ & $258.58^{b}$ & $302.41^{a}$ & $302.18^{a}$ & 12.16 & 0.03 \\
\hline Crypt depth ( $\mu \mathrm{m})$ & 252.26 & 220.42 & 246.12 & 16.26 & 0.37 \\
\hline Villus height/Crypt depth & 1.08 & 1.40 & 1.26 & 0.10 & 0.10 \\
\hline \multicolumn{6}{|l|}{ Jejunum } \\
\hline Villus height ( $\mu \mathrm{m})$ & $271.33^{b}$ & $356.53^{a}$ & $296.00^{b}$ & 11.52 & $<0.01$ \\
\hline Crypt depth ( $\mu \mathrm{m})$ & $200.69^{a}$ & $159.42^{b}$ & $183.48^{a}$ & 7.45 & $<0.01$ \\
\hline Villus height/Crypt depth & $1.37^{b}$ & $2.25^{\mathrm{a}}$ & $1.62^{b}$ & 0.11 & $<0.01$ \\
\hline \multicolumn{6}{|l|}{ Ileum } \\
\hline Villus height ( $\mu \mathrm{m})$ & 255.62 & 291.54 & 264.89 & 11.78 & 0.11 \\
\hline Crypt depth ( $\mu \mathrm{m})$ & 151.08 & 141.07 & 150.93 & 4.97 & 0.29 \\
\hline Villus height/Crypt depth & 1.70 & 2.07 & 1.76 & 0.11 & 0.06 \\
\hline
\end{tabular}

between CON, CTC, and CE groups was presented with PCoA (Figure 1B). The PCoA with the Bray-Curtis distance indicated that the samples of the CON group gathered together and clearly separated from the samples of the CE and CTC groups.

At the phylum level (Figure 2A), the dominant phyla of cecum in three groups were Firmicutes and Proteobacteria. The 
relative abundance of Firmicutes in cecum was significantly increased in piglets fed CE and CTC diets compared with CON diet $(p<0.01)$. Feeding CE and CTC diet significantly decreased the relative abundance of Proteobacteria and Actinobacteriota in cecum $(p<0.01)$. On day 28 , the top three genera in the CON group were Escherichia-Shigella, Lactobacillus, and Succiniclasticum (Figure 2B), while those in CTC group were Lactobacillus, Clostridium_sensu_stricto_1, and Streptococcus, and Lactobacillus, norank_f_T34, and Clostridium_sensu_stricto_1 in CE group. At the genus level, the $\mathrm{CE}$ supplementation significantly decreased the relative abundance of Escherichia_Shigella, Collinsella, and Syntrophococcus in cecum compared with CON $(p<0.01)$. Piglets fed CE diet had greater relative abundance of Lactobacillus than those fed the other two diets $(p<0.001)$. In addition, supplementation of CE and CTC diet reduced $(p<0.05)$ the relative abundance of Succiniclasticum in cecum when compared with the CON diet.

The LEfSe analysis was used to identify the significantly different bacteria at the genus level between the three treatments (Figure 3A). A total of 15 cecal genera were identified to be significantly different between the three groups, namely, 3 genera from CE, 8 genera from CON, and 4 genera from CTC. The relative abundance of g_Lactobacillus, g_Clostridium_sensu_stricto, and g_Terrisporobacter in cecum was increased in piglets fed with the $\mathrm{CE}$ diet; the relative abundance of g_Escherichia-Shigella, g_Succiniclasticum, g_Collinsella, g_Syntrophococcus, g_Bifidobacterium, g_Erysipelotrichaceae_UCG006, g_Ruminococcus_gauvreauii_group, and g_Catenisphaera was increased in piglets fed with the CON diet, whereas the relative abundance of $g_{-}$Streptococcus, g_Treponema, g_UCG005, and g_Lachnospiraceae_XPB1014_group was increased in cecum of piglets fed with the CON diet.

Correlation analysis between serum biochemical parameters and cecal microorganisms has been shown in Figure 3B, wherein the bacteria including g_Lactobacillus, g_Escherichia-Shigella, g_Clostridium_sensu_stricto_1, g_Streptococcus, g_UCG-005, g_Succiniclasticum, g_Collinsella, g_Treponema, g_Bifidobacterium, g_Syntrophococcus, g_Erysipelotrichaceae_UCG006, g_Ruminococcus_gauvreauii group, g_Lachnospiraceae_XPB1014_group, and g_Catenisphaeras had a strong positive correlation with the concentration of $\operatorname{IgG}(p<0.01)$. In addition, the bacteria including g_Lactobacillus and g_Escherichia___Shigellag_Succiniclasticum had a strong positive correlation with the GSH-Px $(p<0.01)$; the bacteria including g_Clostridium_sensu_stricto_1, g_Treponema, and g_Ruminococcus_gauvreauii_group had a strong positive correlation with the MDA $(p<0.01)$.

\section{DISCUSSION}

Adding multi-enzyme in the corn-soybean meal diet not only increased the ADG and G:F, but also increased the ATTD of ADF and NDF in the early and late stages of the experiment. However, dietary treatments had no significant effect on the ATTD of GE and the reason could be that the NSP enzyme acting as fiberdegrading enzyme has greater effects on fiber digestibility in ileum but no other nutrients such as fat and protein digestibility; therefore, no change in ATTD of GE was detected (Li et al., 2018). However, NSP can release more nutrients from the cell wall of grain, while neutral protease can decompose soybean antigen proteins in soybean meal, which are difficult for mammals to decompose into small peptides and amino acids (Meinlschmidt et al., 2016; Munyaka et al., 2016). Since the small peptides and amino acids are easily absorbed, the supplementation of compund enzyme consequently induced improved utilization efficiency of feed and promoted growth of piglets. Our study is consistent with previous studies in that CE promoted the growth of piglets by increasing the conversion rate of nutrients in the feed (Zhang et al., 2014; Li et al., 2018). Tsai et al. (2017) reported that adding both xylanase and $\beta$-glucanase into nursery diets with $30 \%$ corn DDGS improved ADG from days 7 to 35 . However, according to the meta-analysis of Torres-Pitarch et al. (2017), $39 \%$ of studies showed that CE had no effects on pig growth performance, while $61 \%$ of studies showed that CE could improve pig growth performance. There are many factors affecting the growth performance of pigs, such as the proportion of $\mathrm{CE}$, active ingredients, ages and breeds of pigs, feeding environment, and so on. In the future, it will be important to accurately predict the type and concentration of enzymes added to the diet according to different diets and growth stages of pigs.

Immunoglobulins are abundant in serum, have antibody activity or chemical structure similar to antibody molecules, and consist of two identical light and heavy chains linked by disulfide interchain bonds (Schroeder and Cavacini, 2010). Immunoglobulins play an important role in participating in antigen response and improving anti-immune function of weaned piglets. Long et al. (2021) found that CE increased serum immunoglobulins (IgA, IgG, and IgM) of pigs, indicating that $\mathrm{CE}$ could enhance the immune function of weaned pigs. He et al. (2020) reported that the CE could enhance the sIgA content in jejunal mucosa, which indicated that CE could help improve intestinal immune function of weaned pigs. Our study is also consistent with the results of previous studies in that $\mathrm{CE}$ increased serum IgA and IgG levels of piglets, reflecting that $\mathrm{CE}$ can improve the disease resistance of piglets. The CE could probably decompose the NSP in the soybean diet into oligosaccharides, which could help improve the immune function in pigs (Agyekum et al., 2015). The normal growth of animals can be guaranteed by maintaining the relative stability between systematic oxidation and antioxidant status. As the main product of lipid peroxidation, MDA will induce oxidative stress response when it was excessively produced and deposited (Ozdemir et al., 2007). SOD, GSH-Px, and other antioxidant enzymes can use the chain reaction mechanism to reduce the free radical reaction and protect animal from the damage of pro-oxidants (Benzie, 2003; Minelli et al., 2009; Yin et al., 2013). In the present study, dietary CE supplementation showed increased concentrations of antioxidant enzymes including SOD and GSH-Px in serum and decreased serum MDA concentration, which indicated that $\mathrm{CE}$ 

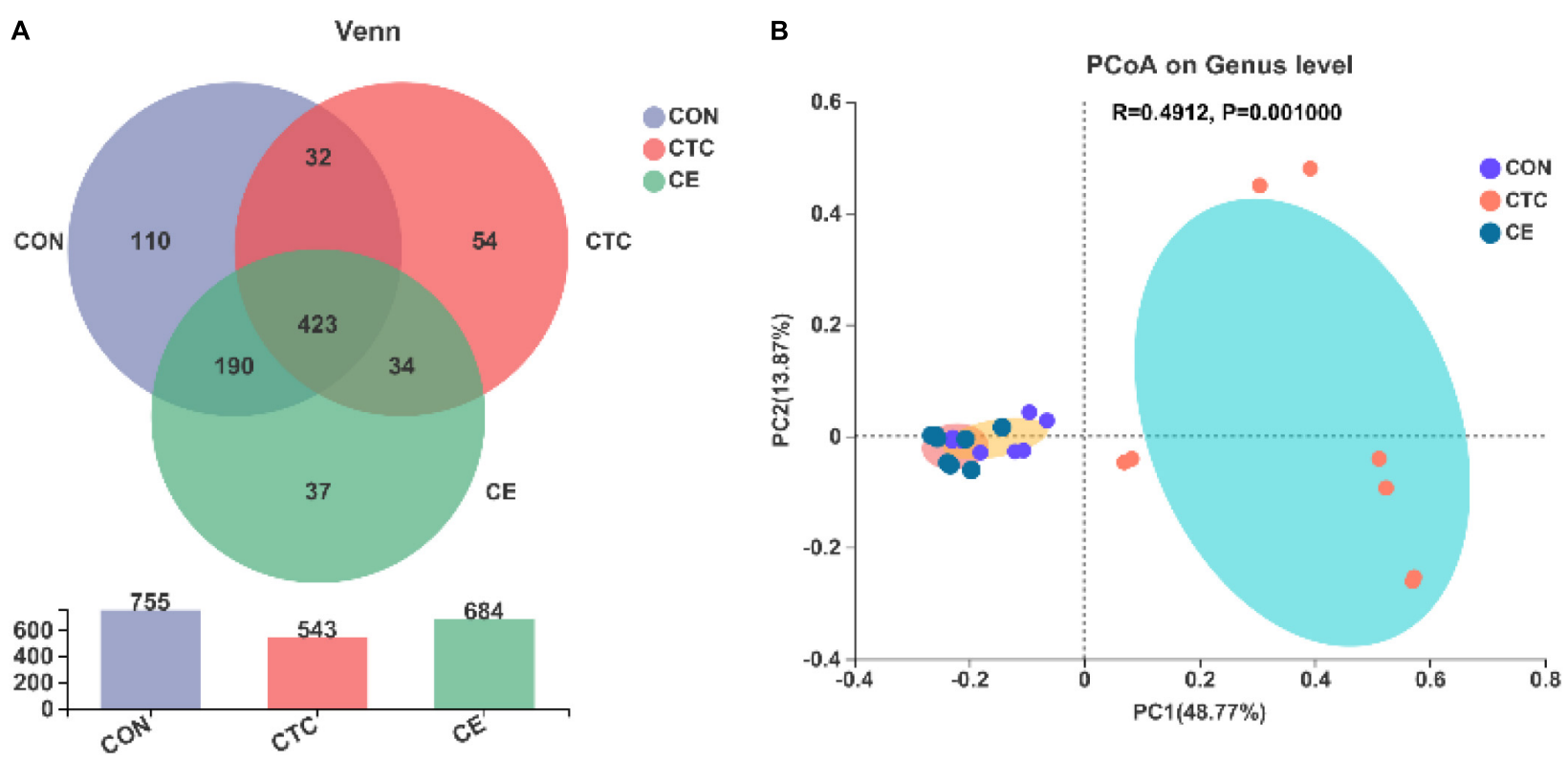

FIGURE 1 | Effect of compund enzymes on cecal OTU of pigs (A). $\beta$-diversity of cecal microbes in piglets (B). The individual minipig was regarded as the experimental unit, $n=8$ for CE, $n=8$ for CON, and $n=8$ for CTC. CE, compund enzyme group; CON, basal diet group; CTC, chlortetracycline group.

may play an important role in eliminating reactive free radicals and alleviating oxidative stress response. This finding was partly consistent with the study of Long et al. (2021) who reported that dietary CE supplementation showed increased serum SOD and CAT content, and reduced serum MDA content. Additionally, Han et al. (2017) found that adding CE containing NSP enzyme and acid protease to piglet diet with antibiotics could significantly increase serum GSH-Px activity and significantly reduce MDA levels. Obviously, the improved antioxidant capacity may lead to reduced damage in intestinal barrier and alleviation of diarrhea (Huang et al., 2015). The positive effects of CE could be explained by reduced anti-nutritional factors in soybean meals due to the neutral protease addition, which may promote the healthy development of the intestinal tract, increase the colonization of beneficial bacteria in the intestinal tract, and reduce the post-intestinal fermentation, thereby improving the antioxidant capacity of piglets.

Microbial fermentation mainly occurs in the cecum and colon of pigs and play an important role in the intestinal health of pigs. It can produce a variety of VFAs, mainly including formic acid, acetic acid, propionic acid, and butyric acid, which can effectively inhibit the reproduction of harmful bacteria and enhance the absorption of intestinal nutrients (Högberg and Lindberg, 2006). In our study, the acetic acid, propionic acid, and total VFA concentrations in cecum and colon were significantly increased in pigs fed the diet supplemented with compound enzyme than those without enzymes. Similarly, Long et al. (2021) reported that dietary CE supplementation tended to increase acetic acid content in colon. Yi et al. (2013) also found that dietary CE supplementation could effectively enhance the VFA contents and improve the health status in weaned pigs. According to the previous studies, acetic acid and propionic acid contents in the large intestine of piglets increased due to dietary CE supplementation; one explanation could be the improved microflora profiles since acetic acid and propionic acid are the main metabolites of microflora in the large intestine (Yi et al., 2013; Zhang et al., 2014). Since VFA has been linked with intestinal health of animals, a higher VFA concentration also explained the improvement in growth performance of piglets.

The integrity of intestinal morphology and structure is a necessary condition to maintain the growth and healthy status of piglets. The ratio of intestinal villus height to crypt depth can directly reflect nutrient digestibility and gastrointestinal absorption function. A higher villus height-to-crypt depth ratio was more favorable for pigs to digest and absorb nutrient ( $\mathrm{Lm}$ et al., 2003). However, weaning stress can significantly reduce intestinal villus height and villus atrophy can lead to intestinal cell death and reduce cell renewal rate (Wang et al., 2011). In our study, supplementation of CE increased the villus height of duodenum and jejunum as well as the villus height-to-crypt depth ratio in ileum. This finding was in agreement with the study of Long et al. (2021), who reported that CE could increase the villus height and the villus height-to-crypt depth ratio in ileum of weaned pigs. Jiang et al. (2015) also found that CE could improve ileal histology and intestinal health of weaning piglets. The reason for the current finding might be that $\mathrm{CE}$ can improve nutrient digestion and absorption in the small intestine and improve the intestinal integrity due to the increased nutrient supply to the intestinal tract (Wijtten et al., 2011).

This study also found that CE increased the relative abundance of Firmicutes and Lactobacillus in cecum, while the relative abundance of Proteobacteria and Actinobacteriota and Escherichia-Shigella were reduced by CE. These results 


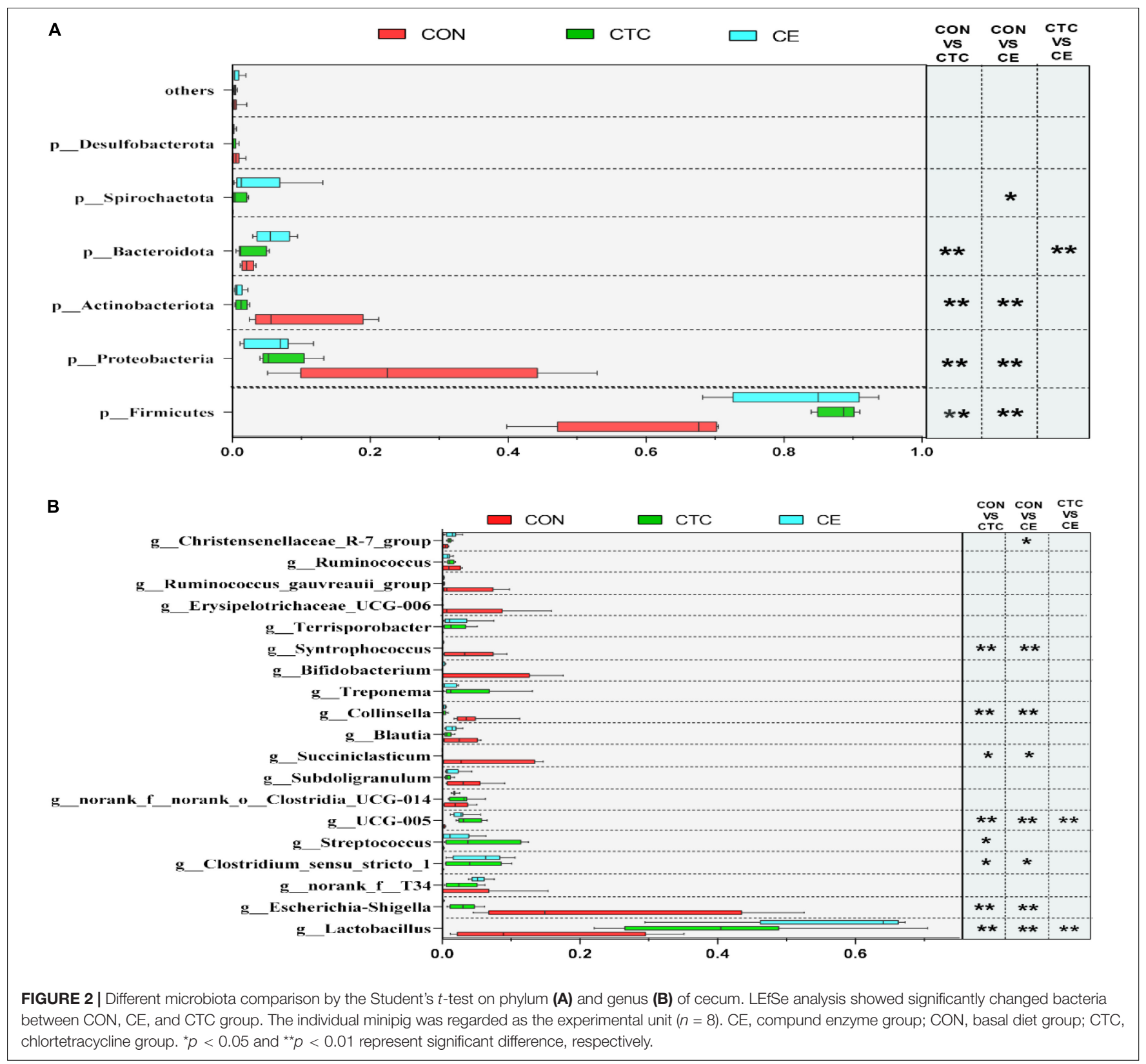

indicated that $\mathrm{CE}$ had a good regulatory effect on the intestinal microflora of piglets. Moreover, an increase in the relative abundance of lactic acid bacteria or a decrease in the relative abundance of Escherichia-Shigella may be more beneficial for the intestinal health of weaned piglets and contributed to an improved growth performance in the CE group. Similar to our results, Long et al. (2021) found that dietary CE supplementation increased the relative abundance of Firmicutes, Bacilli, and Lactobacillus in cecum and colon. The changed microbial profiles induced by dietary treatments could be that the combination of protease, amylase, and cellulase reduced the posterior intestinal fermentation of piglets, inhibited the reproduction of harmful bacteria, and increased the abundance of beneficial bacteria. At the same time, the combination of exogenous amylase or $\beta$-glucanase with other enzymes can also reduce the relative abundance of harmful bacteria such as Escherichia coli in the intestinal tract of weaned piglets, which helps to reduce the diarrhea rate (Zhang et al., 2014; Jiang et al., 2015). Overall, these findings revealed that $\mathrm{CE}$ could be used to replace CTC to achieve a growth-promoting effect in some extent and may be even better than antibiotics in improving intestinal microflora in weaned piglets.

Members of Firmicutes can produce short-chain fatty acids by degrading carbohydrate, which are closely related to energy acquisition and immune response regulation in the body (Atarashi et al., 2011; Zhang et al., 2015). Clostridium has been reported to affect the accumulation of $\mathrm{CD} 8^{+}$IELs (intraepithelial lymphocytes) in the colon (Umesaki et al., 1999). Based on 
A

A LErSe Bar

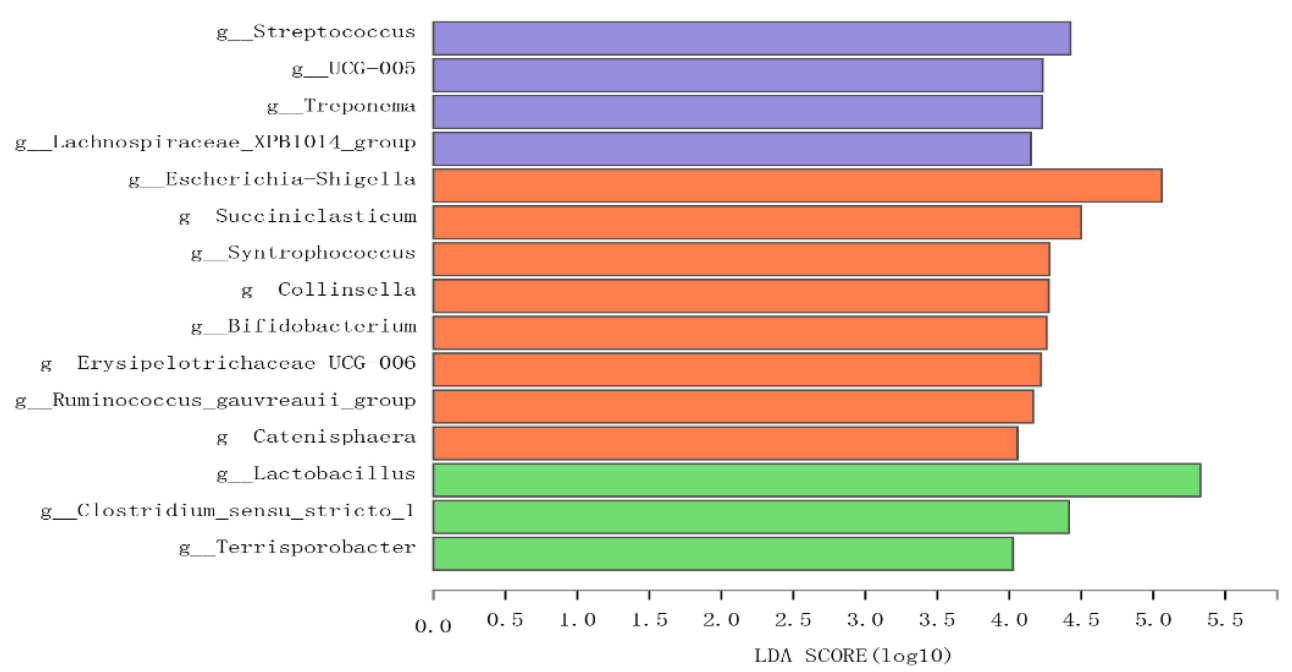

B

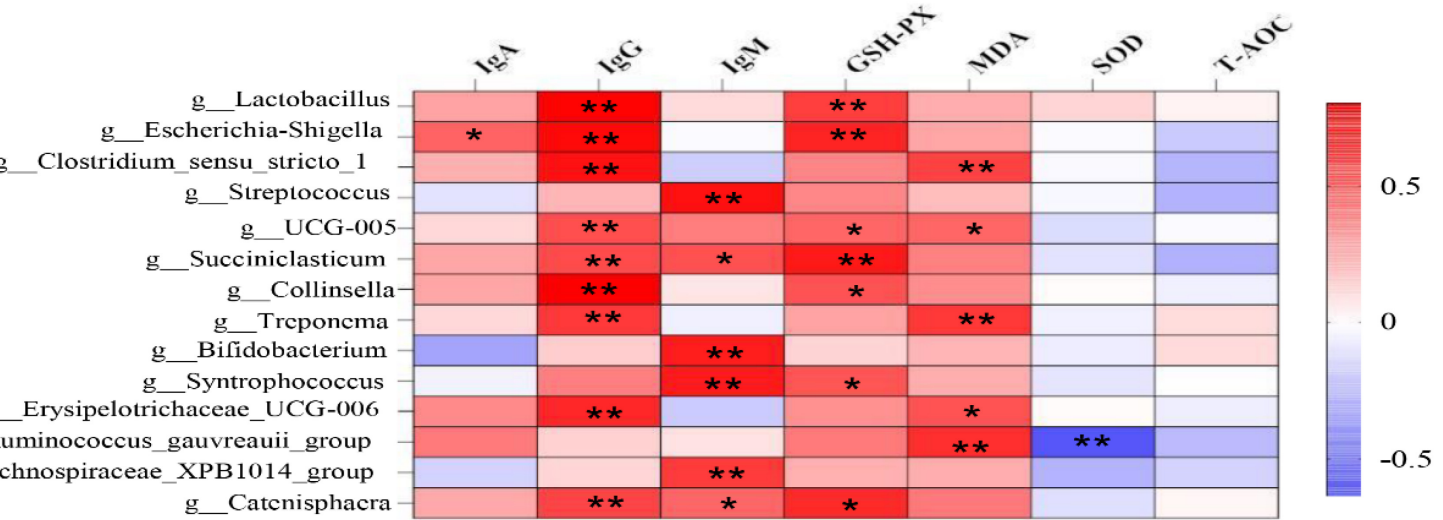

FIGURE 3 | Identification of the most differentially abundant genera in cecum (A). The plot is generated from Linear Discriminant Analysis Effect Size (LEfSe) analysis with CSS-normalized OTU table and displays taxa with LDA scores above 4 and $p$-values below 0.05 . Genera enriched in the samples with CTC diet are indicated with red bars, genera enriched in the samples with CON diet are indicated with blue bars, and genera enriched in the samples with CE diet are indicated with green bars. The individual minipig was regarded as the experimental unit $(n=8)$. CE, compund enzyme group; CON, basal diet group; CTC, chlortetracycline group. Correlation analysis between serum biochemical parameters and cecal microorganisms (B). ${ }^{*} p<0.05$ and ${ }^{* *} p<0.01$ represent significant difference, respectively.

these studies, it is not hard to find that microbes can modulate various aspects of the immune system function. In our study, the correlation analysis between serum biochemical indexes and cecal microorganisms showed that g_Lactobacillus, g_UCG005, g_Succiniclasticum, g_Collinsella, and g_Catenisphaeras had a positive correlation with the concentration of IgG and GSH-Px, while g_Ruminococcus-gauvreauii-group had a negative correlation with the concentration of $\mathrm{MDA}$ and SOD. Some families and genera of Clostridiales cannot only degrade oligosaccharides into butyrate, but also regulate the balance of intestinal microflora (Louis and Flint, 2009) and are mediated by G-protein-coupled receptors in immune cells. Some members of the Lactobacillales, Clostridiales, and Bifidobacteriales are metabolically capable of producing conjugated linoleic acid (Devillard et al., 2007), which has an ability to enhance animal immunity by increasing the activity of immunoglobulin in serum and antioxidant enzymes in liver of animals (Ramírez-Santana et al., 2011; Huang et al., 2017). Besides lactic acid and acetic acid production (Mitsuoka, 1990), Bifidobacterium can promote the production of cytokines, specific antibodies, and non-specific antibodies, which induced activated immunity and enhanced disease resistance (MoyaPérez et al., 2017). Its mechanism of action may be that Bifidobacterium forms a defensive barrier through competitive inhibition, and its metabolites can increase the content of free cholic acid and inhibit the growth of pathogenic microorganisms by activating the body to produce catalase (Zuo et al., 2013). The study of Liu et al. (2017) showed that Lactoales and Enterococcus faecalis could increase the intestinal immune function of piglets and reduce the incidence of diarrhea and mortality of piglets. Similarly, studies have shown that daily probiotic strains containing Bifidobacterium and 
Lactobacillus treatment in mice can alter the inflammatory state of mice (Giacinto et al., 2005; Lyons et al., 2010; Karimi et al., 2012).

\section{CONCLUSION}

In summary, supplementation of CE in diets effectively improved nutrient digestibility and immunity function in weaned piglets and increased relative abundance of Lactobacillus species in the cecum, which could contribute to the improved intestinal health, and consequently was associated with improved growth performance and reduced diarrhea rate of pigs. Overall, CE had comparative effects on growth performance, immunity, antioxidant capacity, and intestinal health of weaned piglets.

\section{DATA AVAILABILITY STATEMENT}

The datasets presented in this study can be found in online repositories. The names of the repository/repositories and accession number(s) can be found below: NCBI SRA database and Bioproject accession number: PRJNA760807 (https://www. ncbi.nlm.nih.gov/bioproject/PRJNA760807).

\section{REFERENCES}

Agyekum, A. K., Sands, J. S., Regassa, A., Kiarie, E., Weihrauch, D., Kim, W. K., et al. (2015). Effect of supplementing a fibrous diet with a xylanase and $\beta$-glucanase blend on growth performance, intestinal glucose uptake, and transport-associated gene expression in growing pigs. J. Anim. Sci. 93, 34833493. doi: $10.2527 /$ jas.2015-9027

Atarashi, K., Tanoue, T., Shima, T., Itoh, Y., and Honda, K. (2011). Induction of colonic regulatory T cells by indigenous Clostridium species. Science (New York, N.Y.) 331, 337-341. doi: 10.1126/science. 1198469

Benzie, I. F. (2003). Evolution of dietary antioxidants. Comp. Biochem. Physiol. Part A Mol. Integr. Physiol. 136, 113-126. doi: 10.1016/s1095-6433(02)00368-9

Chen, S., Zhou, Y., Chen, Y., and Gu, J. (2018). fastp: an ultra-fast all-in-one FASTQ preprocessor. Bioinformatics (Oxford, England) 34, i884-i890. doi: 10. 1093/bioinformatics/bty560

Devillard, E., Mcintosh, F. M., Duncan, S. H., and Wallace, R. J. (2007). Metabolism of linoleic acid by human gut bacteria: different routes for biosynthesis of conjugated linoleic acid. J. Bacteriol. 189, 2566-2570. doi: 10.1128/JB.01359-06

Edgar, R. C. (2013). UPARSE: highly accurate OTU sequences from microbial amplicon reads. Nat. Methods 10, 996-998. doi: 10.1038/nmeth.2604

Giacinto, C. D., Marinaro, M., Sanchez, M., Strober, W., and Boirivant, M. (2005). Probiotics ameliorate recurrent th1-mediated murine colitis by inducing il10 and il-10-dependent tgf- $\beta$-bearing regulatory cells. J. Immunol. 174:3237. doi: 10.4049/jimmunol.174.6.3237

Han, X. Y., Yan, F. Y., Nie, X. Z., Xia, W., Chen, S., Zhang, X. X., et al. (2017). Effect of replacing antibiotics using multi-enzyme preparations on production performance and antioxidant activity in piglets. J. Integr. Agric. 16, 640-647. doi: 10.1016/S2095-3119(16)61425-9

He, X., Yu, B., He, J., Huang, Z., Mao, X., and Zheng, P. (2020). Effects of xylanase on growth performance, nutrients digestibility and intestinal health in weaned piglets - sciencedirect. Livest. Sci. 233:103940.

Högberg, A., and Lindberg, J. E. (2006). The effect of level and type of cereal non-starch polysaccharides on the performance, nutrient utilization and gut environment of pigs around weaning. Anim. Feed Sci. Technol. 127, 200-219. doi: 10.1016/j.anifeedsci.2005.09.004

Huang, C., Song, P., Fan, P., Hou, C., Thacker, P., and Ma, X. (2015). Dietary sodium butyrate decreases postweaning diarrhea by modulating intestinal

\section{ETHICS STATEMENT}

The animal study was reviewed and approved by the Committee of Animal Care at Hunan Agricultural University (Changsha, China) (Permit Number: CACAHU 2021-01106).

\section{AUTHOR CONTRIBUTIONS}

ZL and XM: conceptualization, methodology, and software. LT, NL, XL, and FZ: literature collection. ZL and QJ: writingoriginal draft preparation. JC: writing-reviewing and editing. XM: funding acquisition. All authors contributed to the article and approved the submitted version.

\section{FUNDING}

This review was funded by the Scientific Research Fund of Hunan Provincial Education Department (19B267), the Youth Science Foundation Project of Hunan Agricultural University (19QN01), and the Open Foundation of CAS Key Laboratory of Agro-ecological Processes in Subtropical Region, Institute of Subtropical Agriculture (ISA2020101).

permeability and changing the bacterial communities in weaned piglets. J. Nutr. 145, 2774-2780. doi: 10.3945/jn.115.217406

Huang, Y. S., Lin, Z. D., Rong, H., Hao, M. L., Zhu, D. S., Li, S. K., et al. (2017). Effects of conjugated linoleic acid on growth, body composition, antioxidant status, lipid metabolism and immunity parameters of juvenile chu's croaker, nibea coibor. Aquacul. Res. 49, 546-556. doi: 10.1111/are.13486

Jiang, X. R., Awati, A., Agazzi, A., Vitari, F., Ferrari, A., Bento, H., et al. (2015). Effects of a blend of essential oils and an enzyme combination on nutrient digestibility, ileum histology and expression of inflammatory mediators in weaned piglets. Animal 9, 417-426. doi: 10.1017/S175173111400 2444

Karimi, K., Inman, M. D., Bienenstock, J., and Forsythe, P. (2012). Lactobacillus reuteri-induced regulatory t cells protect against an allergic airway response in mice. Am. J. Respir. Crit. Care Med. 179, 186-193. doi: 10.1164/rccm.200806$9510 \mathrm{C}$

Li, H., Yin, J., He, X., Li, Z., Tan, B., Jiang, Q., et al. (2021). Enzyme-treated soybean meal replacing extruded full-fat soybean affects nitrogen digestibility, cecal fermentation characteristics and bacterial community of newly weaned piglets. Front. Vet. Sci. 8:639039. doi: 10.3389/fvets.2021.639039

Li, Q., Gabler, N. K., Loving, C. L., Gould, S. A., and Patience, J. F. (2018). A dietary carbohydrase blend improved intestinal barrier function and growth rate in weaned pigs fed higher fiber diets. J. Anim. Sci. 96, 5233-5243. doi: $10.1093 /$ jas/sky383

Liu, C., Zhu, Q., Chang, J., Yin, Q., Song, A., Li, Z., et al. (2017). Effects of Lactobacillus casei and enterococcus faecalis on growth performance, immune function and gut microbiota of suckling piglets. Arch. Anim. Nutr. 71, 120-133. doi: 10.1080/1745039X.2017.1283824

Lm, A., Jrp, B., and Djh, B. (2003). A review of interactions between dietary fibre and the intestinal mucosa, and their consequences on digestive health in young non-ruminant animals - sciencedirect. Anim. Feed Sci. Technol. 108, 95-117.

Long, S., Hu, J., Mahfuz, S., Ma, H., and Piao, X. (2021). Effects of dietary supplementation of compound enzymes on performance, nutrient digestibility, serum antioxidant status, immunoglobulins, intestinal morphology and microbiota community in weaned pigs. Arch. Anim. Nut. 75, 31-47. doi: 10. 1080/1745039X.2020.1852008

Long, S. F., Xu, Y. T., Pan, L., Wang, Q. Q., Wang, C. L., Wu, J. Y., et al. (2017). Mixed organic acids as antibiotic substitutes improve performance, serum 
immunity, intestinal morphology and microbiota for weaned piglets. Anim. Feed Sci. Technol. 235, 23-32. doi: 10.1016/j.anifeedsci.2017.08.018

Louis, P., and Flint, H. J. (2009). Diversity, metabolism and microbial ecology of butyrate-producing bacteria from the human large intestine. FEMS Microbiol. Lett. 294, 1-8. doi: 10.1111/j.1574-6968.2009.01514.x

Lyons, A., O’Mahony, D., O’Brien, F., Macsharry, J., and O’Mahony, L. (2010). Bacterial strain-specific induction of foxp3+t regulatory cells is protective in murine allergy models. Clin. Exp. Allergy 40, 811-819. doi: 10.1111/j.1365-2222. 2009.03437.x

Magoč, T., and Salzberg, S. L. (2011). FLASH: fast length adjustment of short reads to improve genome assemblies. Bioinformatics 27, 2957-2963. doi: 10. 1093/bioinformatics/btr507

Meinlschmidt, P., Schweigee, U., and Rode, V. (2016). Enzyme assisted degradation of po-tential soy protein allergens with special emphasis onthe technofunctionality and the avoidance of a bitter taste formation. LWT Food Sci. Technol. 68, 707-716. doi: 10.2767/jas.2000-5598

Minelli, A., Bellezza, I., Conte, C., and Culig, Z. (2009). Oxidative stress-related aging: a role for prostate cancer? Biochim. Biophys. Acta 1795, 83-91. doi: 10.1016/j.bbcan.2008.11.001

Mitsuoka, T. (1990). Bifidobacteria and their role in human health. J. Ind. Microbiol. 6, 263-267. doi: 10.1007/BF01575871

Moya-Pérez, A., Perez-Villalba, A., Benítez-Páez, A., Campillo, I., and Sanz, Y. (2017). Bifidobacterium cect 7765 modulates early stress-induced immune, neuroendocrine and behavioral alterations in mice. Brain Behav. Immun. 65, 43-56. doi: 10.1016/j.bbi.2017.05.011

Munyaka, P. M., Nandha, N. K., Kiarie, E., Nyachoti, C. M., and Khafipour, E. (2016). Impact of combined $\beta$-glucanase and xylanase enzymes on growth performance, nutrients utilization and gut microbiota in broiler chickens fed corn or wheat-based diets. Poult. Sci. 95, 528-540. doi: 10.3382/ps/pev333

NRC (2012). Nutrient Requirements of Swine, 11th Edn. Washington, DC: National Academies Press.

Ozdemir, D., Uysal, N., Tugyan, K., Gonenc, S., Acikgoz, O., Aksu, I., et al. (2007). The effect of melatonin on endotoxemia-induced intestinal apoptosis and oxidative stress in infant rats. Intensive Care Med. 33, 511-516. doi: 10. 1007/s00134-006-0492-z

Ramírez-Santana, C., Castellote, C., Castell, M., Moltó-Puigmartí, C., Rivero, M., Pérez-Cano, F. J., et al. (2011). Enhancement of antibody synthesis in rats by feeding cis-9,trans-11 conjugated linoleic acid during early life. J. Nutr. Biochem. 22, 495-501. doi: 10.1016/j.jnutbio.2010.04.005

Schroeder, H. W. Jr., and Cavacini, L. (2010). Structure and function of immunoglobulins. J. Allergy Clin. Immunol. 125(2 Suppl 2), S41-S52.

Teale, C., and Borriello, P. (2021). A proposed scheme for the monitoring of antibiotic resistance in veterinary pathogens of food animals in the uk. Vet. Rec. 189:e201. doi: 10.1002/vetr.201

Torres-Pitarch, A., Hermans, D., Manzanilla, E. G., Bindelle, J., Everaert, N., Beckers, Y., et al. (2017). Effect of feed enzymes on digestibility and growth in weaned pigs: a systematic review and meta-analysis. Anim. Feed Sci. Technol. 233, 145-159. doi: 10.1016/j.anifeedsci.2017.04.024

Tsai, T., Dove, C. R., Cline, P. M., Owusu-Asiedu, A., Walsh, M. C., and Azain, M. (2017). The effect of adding xylanase or $\beta$-glucanase to diets with corn distillers dried grains with solubles (cddgs) on growth performance and nutrient digestibility in nursery pigs. Livest. Sci. 197, 46-52. doi: 10.1016/j.livsci. 2017.01.008

Umesaki, Y., Setoyama, H., Matsumoto, S., Imaoka, A., and Itoh, K. (1999). Differential roles of segmented filamentous bacteria and clostridia in development of the intestinal immune system. Infect. Immun. 67:3504. doi: 10.1111/j.1574-695X.1999.tb01309.x

Vila, M. F., Trudeau, M. P., Hung, Y. T., Zeng, Z., Urriola, P. E., Shurson, G. C., et al. (2018). Dietary fiber sources and non-starch polysaccharide-degrading enzymes modify mucin expression and the immune profile of the swine ileum. PLoS One 13:e0207196. doi: 10.1371/journal.pone.0207196

Wang, D., Piao, X. S., Zeng, Z. K., Lu, T., Zhang, Q., Li, P. F., et al. (2011). Effects of keratinase on performance, nutrient utilization, intestinal morphology, intestinal ecology and inflammatory response of weaned piglets fed diets with different levels of crude protein. Asian Australas. J. Anim. Sci. 24, 1718-1728. doi: 10.5713/ajas.2011.11132

Wang, F., Yin, Y., Yang, M., Chen, J., Fu, C., and Huang, K. (2021). Effects of combined supplementation of Macleaya cordata extract and benzoic acid on the growth performance, immune responses, antioxidant capacity, intestinal morphology, and microbial composition in weaned piglets. Front. Vet. Sci. 8:708597. doi: 10.3389/fvets.2021.708597

Wijtten, P. J., van der Meulen, J., and Verstegen, M. W. (2011). Intestinal barrier function and absorption in pigs after weaning: a review. Br. J. Nutr. 105, 967-981. doi: 10.1017/S0007114510005660

Willamil, J., Badiola, I., Devillard, E., Geraert, P. A., and Torrallardona, D. (2012). Wheat-barley-rye- or corn-fed growing pigs respond differently to dietary supplementation with a carbohydrase complex. J. Anim. Sci. 90, 824-832. doi: 10.2527/jas.2010-3766

Woyengo, T. A., Cowieson, A. J., Adeola, O., and Nyachoti, C. M. (2009). Ileal digestibility and endogenous flow of minerals and amino acids: responses to dietary phytic acid in piglets. Br. J. Nutr. 102, 428-433. doi: 10.1017/ S0007114508184719

Yi, J. Q., Piao, X. S., Li, Z. C., Zhang, H. Y., and Dong, B. (2013). The effects of enzyme complex on performance, intestinal health and nutrient digestibility of weaned pigs. Asian Australas. J. Anim. Sci. 26, 1181-1188. doi: 10.5713/ajas. 2013.13129

Yin, J., Ren, W., Wu, X., Yang, G., and Su, D. (2013). Oxidative stress-mediated signaling pathways: a review. J. Food Agric. Environ. 11, 132-139.

Zaefarian, F., Abdollahi, M. R., and Ravindran, V. (2015). Starch digestion in broiler chickens fed cereal diets. Anim. Feed Sci. Technol. 209, 16-29. doi: 10.1016/j.anifeedsci.2015.07.020

Zainab, S. M., Junaid, M., Xu, N., and Malik, R. N. (2020). Antibiotics and antibiotic resistant genes (ARGs) in groundwater: a global review on dissemination, sources, interactions, environmental and human health risks. Water Res. 187:116455. doi: 10.1016/j.watres.2020.116455

Zhang, G. G., Yang, Z. B., Wang, Y., Yang, W. R., and Zhou, H. J. (2014). Effects of dietary supplementation of multi-enzyme on growth performance, nutrient digestibility, small intestinal digestive enzyme activities, and large intestinal selected microbiota in weanling pigs. J. Anim. Sci. 92, 2063-2069. doi: 10.2527/ jas.2013-6672

Zhang, J., Guo, Z., Xue, Z., Sun, Z., Zhang, M., Wang, L., et al. (2015). A phylofunctional core of gut microbiota in healthy young chinese cohorts across lifestyles, geography and ethnicities. ISME J. Multidiscip. J. Microb. Ecol. 9, 1979-1990. doi: 10.1038/ismej.2015.11

Zuo, Q. R., Mai, K., and Xu, W. (2013). Effects of Conjugated Linoleic Acid on Growth, Non-Specific Immunity, Antioxidant Capacity, Lipid Deposition And Related Gene Expression in Juvenile Large Yellow Croaker. Cambridge: Cambridge University Press.

Conflict of Interest: The authors declare that the research was conducted in the absence of any commercial or financial relationships that could be construed as a potential conflict of interest.

Publisher's Note: All claims expressed in this article are solely those of the authors and do not necessarily represent those of their affiliated organizations, or those of the publisher, the editors and the reviewers. Any product that may be evaluated in this article, or claim that may be made by its manufacturer, is not guaranteed or endorsed by the publisher.

Copyright (C) 2021 Li, Tang, Liu, Zhang, Liu, Jiang, Chen and Ma. This is an openaccess article distributed under the terms of the Creative Commons Attribution License (CC BY). The use, distribution or reproduction in other forums is permitted, provided the original author(s) and the copyright owner(s) are credited and that the original publication in this journal is cited, in accordance with accepted academic practice. No use, distribution or reproduction is permitted which does not comply with these terms. 